\title{
Deep lamellar keratoplasty on air with lyophilised tissue
}

G K Chau, S A Dilly, C E Sheard, C K Rostron

\begin{abstract}
Deep lamellar keratoplasty on air involves injecting air into the corneal stroma to expand it to several times its normal thickness. This method is designed to facilitate dissection of the deep stroma and reduce the risk of perforation of Descemet's membrane when carrying out deep lamellar keratoplasty. We have modified the technique by using prelathed freeze-dried donor tissue and report our results in a series of patients with corneal stromal scarring owing to a variety of corneal problems, namely, keratoconus, pterygium, and herpes zoster ophthalmicus. All patients achieved best corrected postoperative visual acuity of $6 / 12$ or better without problems associated with graft failure or rejection. Histopathological examination of corneal tissue following air injection showed surgical emphysema within the cornea and separation of deep stromal fibres from the underlying Descemet's membrane.
\end{abstract}

(Brf Ophthalmol 1992; 76: 646-650)

The technique of deep lamellar keratoplasty on air was first reported by Archila in 1985 in a study of patients with a range of corneal disorders where there was central stromal scarring but no endothelial damage. ${ }^{1}$ The surgical procedure involves injecting air into the corneal stroma, which then expands and becomes temporarily opaque. The deep stromal fibres become separated from Descemet's membrane and this allows a uniform, deep lamellar dissection to be made which is more clearly visualised because of the temporary opacification of the stroma.

Archila reported using donor corneal tissue of full thickness, whereas Price, in a subsequent study, ${ }^{2}$ employed tissue with the endothelium removed. It is recognised that if the endothelium is left on the donor tissue in lamellar keratoplasty it does not survive. ${ }^{3}$ Leaving Descemet's membrane intact on the donor tissue is said to give a better optical surface at the interface in lamellar keratoplasty, ${ }^{4}$ but histological studies of such grafts show little evidence of healing between the donor Descemet's membrane and underlying stroma, giving reduced wound strength and predisposing to subgraft clefts and even pseudoanterior chambers. ${ }^{5}$ Histological examination of the effect of air injection into corneal stroma has also been carried out in animal eyes by Jongebloed et al. ${ }^{6}$ They performed scanning electron microscopy on sections of porcine corneas which had previously been injected with air and reported the finding of stromal canals.

Lamellar keratoplasty can be used for the treatment of corneal diseases in which the endothelium is healthy, as in keratoconus, some

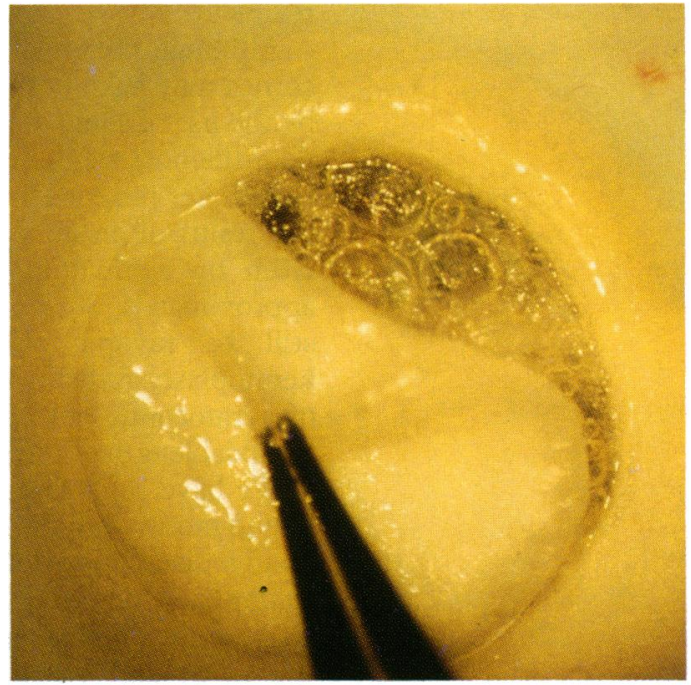

Figure 1 Complete air dissection of the cornea in a cadaver globe. Lamellar dissection is being carried out near Descemet's membrane, and air can be seen in the anterior chamber.

corneal stromal dystrophies, and stromal scarring. However, when compared with penetrating keratoplasty, its advantages must be weighed against its greater technical difficulty, increased operating time, and generally inferior visual outcome. ${ }^{7}$ The advantage of lamellar keratoplasty is that it is free from the problems of corneal decompensation owing to poor donor endothelium, since the graft is reliant on the host endothelial cells. It is also recognised that failure of a lamellar graft from rejection is unlikely, whereas in penetrating grafts immunological allograft rejection is the most common cause of late failure. ${ }^{8}$ It has been demonstrating that graft epithelium, stromal keratocytes, and endothelium can separately undergo immunological rejection, ${ }^{910}$ but epithelial rejection is commonly asymptomatic since the graft is resurfaced by host epithelium as the rejection line advances across the corneal surface." In endothelial rejection however, damage to the endothelial cells may compromise the regulation of hydration of the graft, giving bullous keratopathy, since the human endothelium does not replicate readily. ${ }^{12} 13$

In lamellar keratorefractive procedures, such as epikeratophakia, the donor tissue is often freeze-dried as the cellular viability of the tissue is not essential. ${ }^{14}$ This simplifies tissue preservation and distribution. In addition, it has been shown that experimental fresh tissue epikeratophakia grafts implanted onto a vascularised bed, in a pre-sensitised recipient, may be rejected, whereas freeze-dried epikeratophakia lenticules are not. ${ }^{15}$ The reduced immunogenicity of lyophilised lenticules may be an important factor 

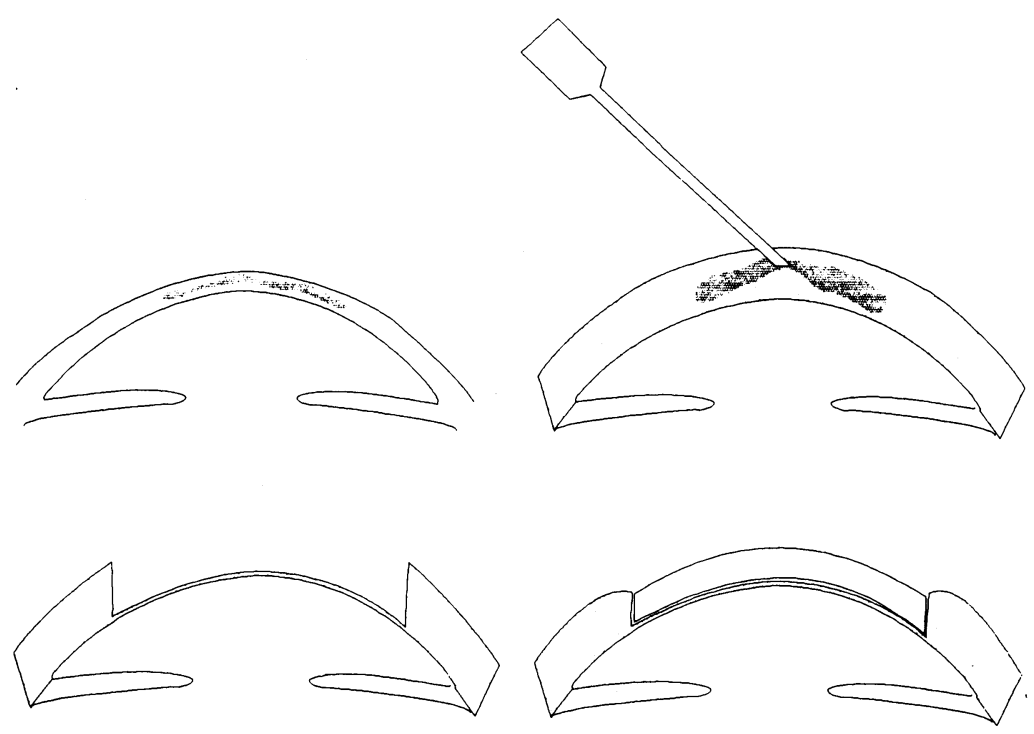

Figure 2 A patient's cornea with central stromal scarring is suitable for this technique. Air is injected and a lamella resected. Lyophilised tissue is sutured in place.

if the recipient patient subsequently were to undergo penetrating keratoplasty since the eye would not be pre-sensitised to donor antigens.

We present our experimental work on human cadaver corneas and the results of using the technique in a series of four patients with different corneal pathologies. The corneal material we used for grafting included both organ-cultured full thickness corneal tissue without endothelium, and lathed lyophilised lenticules.

\section{Materials and methods}

IN VITRO WORK

We initially carried out trials in six human cadaver corneas which did not have pre-existing corneal pathology. Two were simply injected with air, and in the others air was injected and experimental lamellar dissection carried out (Fig 1). These eyes were fixed in formalin and then

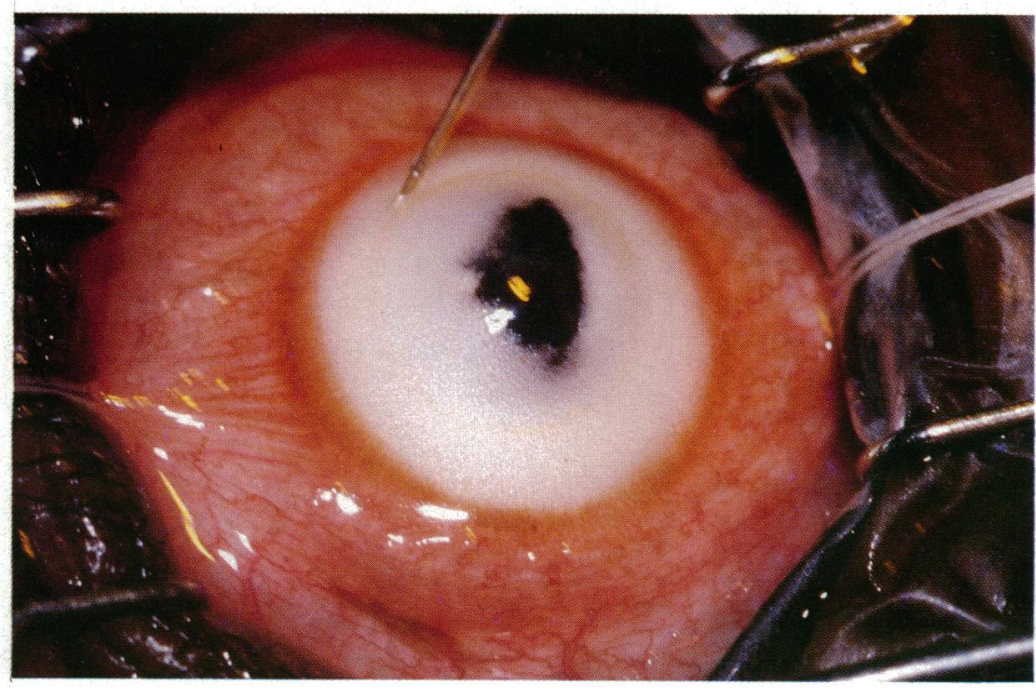

Figure 3 Air injection of patient number 1 (keratoconus). The air has not reached the central zone where there is stromal scarring. Fleischer's ring is highlighted against the opaque insufflated cornea.

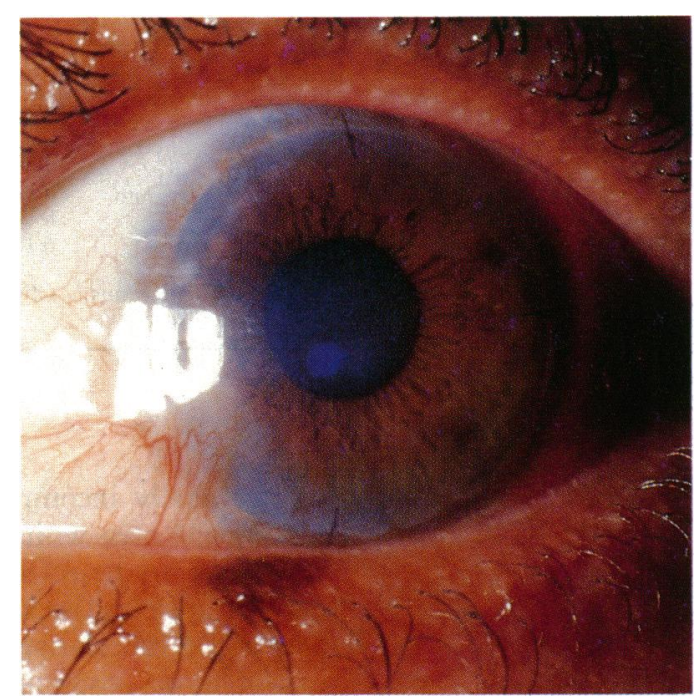

Figure 4 Patient number 2 (pterygium) 6 months postoperatively. The graft used was a $9.5 \mathrm{~mm}$ lathed lyophilised lenticule of $100 \%$ donor central corneal thickness.

slices were paraffin embedded and sections of $3 \mu \mathrm{m}$ cut prior to staining with haematoxylin and eosin for assessment by light microscopy.

\section{CLINICAL SERIES}

The four clinical cases selected all had superficial stromal opacities over the visual axis. Two had moderate keratoconus with significant apical scarring rendering them unsuitable for epikeratoplasty, and both were intolerant of contact lenses. The third patient had extensive vascularised subepithelial scarring following herpes zoster ophthalmicus. In this case the corneal new vessels were shut down with an argon laser preoperatively. The remaining patient had a large nasal pterygium with subepithelial scarring extending across the visual axis.

Surgery was performed by one surgeon (CKR) using a method similar to that described by Archila. A 26 gauge needle was inserted obliquely into the stroma in the mid-periphery of the cornea and air was injected until the whole stroma became insufflated if this was possible. Superficial trephination was made and the incision deepened with a diamond knife until Descemet's membrane was approached. Lamellar stromal dissection was carried out using a disposable lamellar blade. Out of the four cases, one patient (number 1 ) received a tissuecultured full thickness donor cornea which had its endothelium removed by cellulose sponge. The other three patients received pre-lathed lyophilised lenticules cut to $100 \%$ of the donor tissue central corneal thickness (plano lamellar tissue, Keratec Eye Bank, St George's Hospital, London, UK). These lenticules were rehydrated with balanced salt solution just prior to grafting. The grafts were secured with interrupted monofilament sutures (Fig 2).

\section{Results}

Though complete insufflation of the cornea by air injection was relatively easy in cadaver eyes, it 
Table 1 Patient data

\begin{tabular}{|c|c|c|c|c|c|c|c|c|}
\hline $\begin{array}{l}\text { Patient } \\
\text { number }\end{array}$ & Diagnosis & $\begin{array}{l}\text { Best corrected } \\
\text { pre-op acuity }\end{array}$ & $\begin{array}{l}\text { Best corrected } \\
\text { post-op acuity }\end{array}$ & $\begin{array}{l}\text { Pre-op } \\
\text { refraction }\end{array}$ & $\begin{array}{l}\text { Post-op } \\
\text { refraction }\end{array}$ & $\begin{array}{l}\text { Pre-op } \\
\text { keratometry }\end{array}$ & $\begin{array}{l}\text { Post-op } \\
\text { keratometry }\end{array}$ & Comment \\
\hline 1 & Keratoconus & $6 / 18$ & $6 / 12$ & N/A & $\begin{array}{l}-4.0 \mathrm{Sph} \\
-3.5 \times 20^{\circ}\end{array}$ & $\begin{array}{l}\text { Irregular } \\
<5 \cdot 5\end{array}$ & \multirow{4}{*}{$\begin{array}{l}7.78 \text { at } 90^{\circ} \\
7.35 \text { at } 180^{\circ} \\
7.05 \text { at } 60^{\circ} \\
7.98 \text { at } 150^{\circ} \\
7.69 \text { at } 90^{\circ} \\
7.66 \text { at } 180^{\circ} \\
7.7 \text { at } 40^{\circ} \\
6.7 \text { at } 130^{\circ}\end{array}$} & Fresh tissue graft \\
\hline 2 & Pterygium & $6 / 18$ & $6 / 12$ & $\begin{array}{l}+1.5 \mathrm{Sph} \\
-1.75 \times 25^{\circ}\end{array}$ & $\begin{array}{l}+2.5 \mathrm{Sph} \\
-5.0 \times 155^{\circ}\end{array}$ & Irregular & & \multirow{3}{*}{$\begin{array}{l}\text { Epithelial islands } \\
\text { at interface } \\
\text { Epithelial islands } \\
\text { at interface } \\
\text { No complications }\end{array}$} \\
\hline 3 & $\begin{array}{l}\text { Herpes } \\
\text { zoster }\end{array}$ & $6 / 18$ & $6 / 12$ & $\begin{array}{l}+0.5 \mathrm{Sph} \\
+1.0 \times 90^{\circ}\end{array}$ & $+1.75 \mathrm{Sph}$ & Irregular & & \\
\hline 4 & Keratoconus & CF & $6 / 9$ & NA & $\begin{array}{l}-8.5 \mathrm{Sph} \\
+5.0 \times 120^{\circ}\end{array}$ & $\begin{array}{l}\text { Irregular } \\
<5 \cdot 5\end{array}$ & & \\
\hline
\end{tabular}

was generally incomplete in our clinical cases leaving the area with most stromal scarring or pathology undissected. This occurred at the corneal apex in the keratoconus cases (Fig 3), and in the vascularised peripheral sector of the case of herpes zoster ophthalmicus. Complete air dissection of the cornea was achieved only in the case with the pterygium.

In every case air was found to enter the anterior chamber and, to a varying extent, to track into the subconjunctival tissues. In some instances it was necessary to aspirate the air from the anterior chamber by limbal puncture in order to reduce the intraocular pressure, and it was these problems that prevented complete insufflation of the cornea.

Table 1 shows the results of the clinical cases including the preoperative and postoperative visual acuity, refraction, and keratometric values over the follow-up period of up to 1 year.

The first patient with keratoconus only had a planned $50 \%$ lamellar dissection, and postoperatively there was some diffuse interface scarring with a contact lens corrected vision of $6 / 12$. Because the patient was dissatisfied with the visual result he later underwent penetrating keratoplasty.

The second patient who had resection of a nasal pterygium developed small but persistent epithelial defects in the first postoperative month; these healed eventually. Additionally there were three small epithelial islands left at the interface away from the visual axis (Fig 4).

The third patient with vascularised scarring secondary to herpes zoster ophthalmicus had previously undergone extracapsular cataract extraction with posterior chamber lens implantation. Incomplete air dissection was a problem particularly in the area of neovascularisation, and ultimately some deep stromal opacity remained, but this was away from the visual axis.

Patient number four with keratoconus could not be fitted with contact lenses preoperatively; there was obvious central stromal opacity and acuity was counting fingers only. Postoperatively he achieved marked visual improvement to $6 / 9$ with a contact lens. He had no significant complications.

Preoperative spectacle corrected vision ranged between 6/12 and counting fingers. All four patients achieved best corrected vision of $6 / 12$ or better 1 year postoperatively. Considerable postoperative astigmatism was common in the early postoperative phase but tended to settle with time and selective suture removal. Both patients with keratoconus had marked corneal flattening after surgery.

\section{Histopathology}

\section{IN VITRO WORK}

Figures 5 and 6, stained with haematoxylin and eosin, show sections of human cadaver cornea following air insufflation. Nearly the whole stroma is occupied by small oval holes which are smaller superficially but become larger towards Descemet's membrane. The surgical emphysema of the cornea halts abruptly at the corneoscleral junction adjacent to the trabecular meshwork. The fine stromal structure immediately beneath Bowman's layer appears relatively unaffected, while there is good separation of Descemet's membrane from deep stromal fibres. In no specimen was rupture of Descemet's membrane seen, although the possibility that rupture had occurred in a plane different from that of the sections examined was not excluded.

\section{CLINICAL SPECIMENS}

Histological examination of the excised lamellar dissections from the clinical cases revealed a

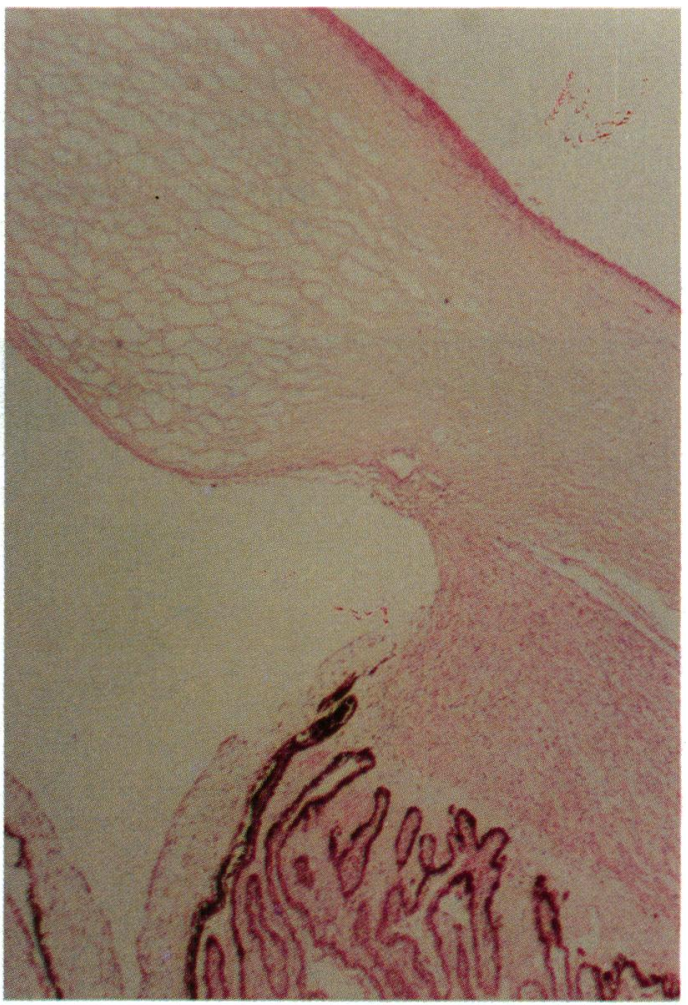

Figure 5 Cadaver cornea following air injection. The stroma is expanded by air spaces which extend only as far as the limbus (haematoxylin and eosin, original magnification $\times 10$ ). 


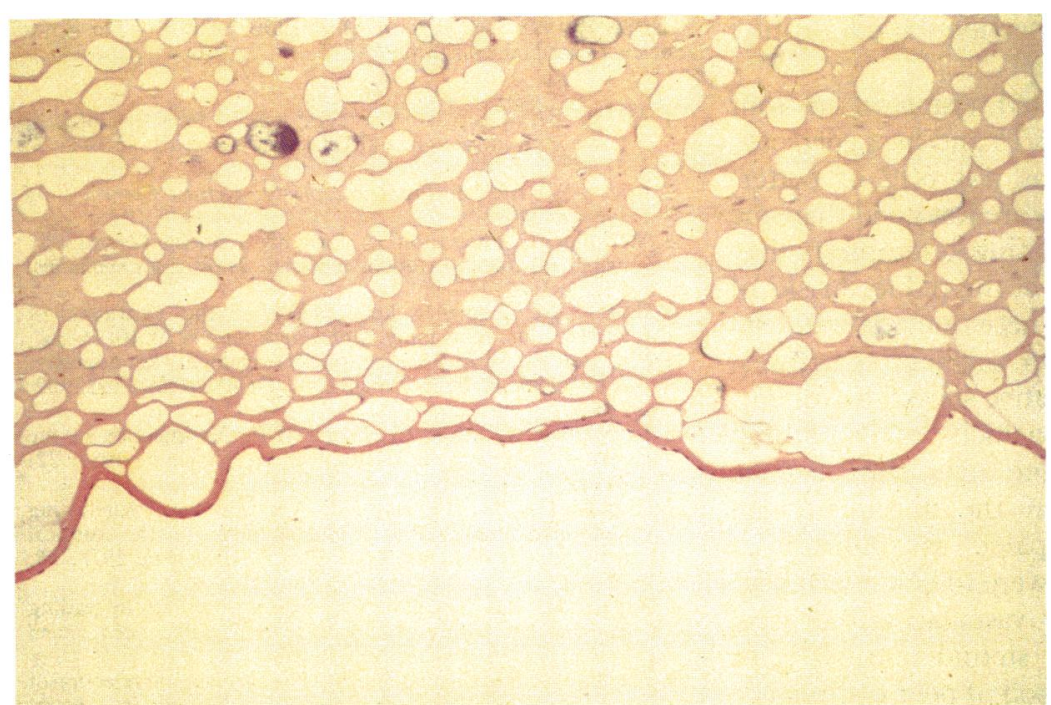

Figure 6 High power view of air dissected cadaver cornea showing separation of Descemet's membrane from deep stromal fibre bundles (haematoxylin and eosin, original magnification $\times 25)$.

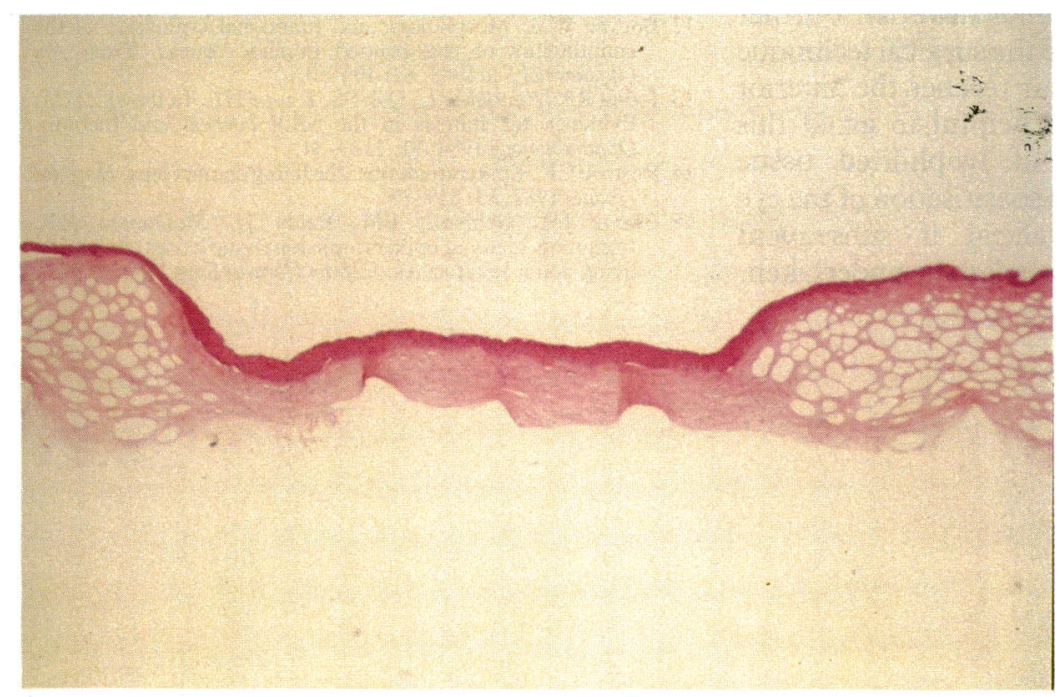

Figure 7 Histopathology of excised corneal lamella from patient number 1 (keratoconus). There is no air dissection of the central scarred zone, but the tissue is expanded on either side (haematoxylin and eosin, original magnification $\times 4$ ).

similar appearance to the cadaver corneas. Figure 7 shows that air penetration is incomplete in this keratoconus specimen where the central stroma with apical scarring is not inflated by air. Though there is some degree of pre-existing central corneal thinning one can appreciate the marked increase of overall stromal thickness caused by air injection. Our histological sections do not reveal any difference in the epithelial layer overlying injected and non-injected areas, at least under light microscopy.

\section{Discussion}

The standard technique for lamellar keratoplasty is to resect the superficial host stroma and to replace the resected tissue with a partial thickness donor lamella. Problems with postoperative interface scarring have caused the technique to be largely discarded in favour of penetrating keratoplasty, which nowadays has a high success rate thanks to modern techniques of endothelial assessment of the donor corneas, and improved tissue handling by microsurgery. Nevertheless it must be remembered that in some conditions for example, after trauma or ulceration, there may be vascularisation of the recipient cornea, and in these circumstances penetrating keratoplasty is not so successful because rejection problems are more frequently encountered.

Deep lamellar keratoplasty on air provides an enhanced visual contrast of the stroma to be dissected, allowing a more accurate assessment of the depth of incision. This helps to achieve resection of as much deep stroma as possible without perforating Descemet's membrane. In practice, dissection actually at the level of Descemet's membrane still carries a high risk of perforation, either through direct incision through the membrane, or by splitting or tearing of the membrane from indirect forces applied to it.

Because of this risk, in none of our clinical cases did we attempt to resect the full thickness of the recipient stroma, but rather aimed to achieve a near full thickness resection in the central optical zone of the cornea, but a less than full thickness resection in the periphery of the bed where the cornea is thicker. By limiting the near full thickness dissection to the central area alone it was thought that the risk of perforation would be minimised, and Descemet's membrane was not in fact ruptured in any of the clinical cases.

By resecting less than the full thickness of the recipient cornea in the periphery of the graft bed, the risk of perforation by direct downward cutting with the diamond knife is minimised. The resultant graft bed thus has a contour that lies a uniform distance from the original corneal surface. In order to match the shape of this resected bed, the donor tissue used for the latter three patients in the series was pre-lathed to a uniform $100 \%$ of the donor tissue's central thickness.

Air dissection was incomplete in each of our patients with stromal scarring, particularly in the area of maximal scarring. It is interesting to note that in cadaver corneas, air dissection is much more easily and quickly achieved than in living tissue, possibly owing to reduced adhesion between adjacent stromal fibre bundles from pre-existing corneal oedema.

We observed air in the anterior chamber in every case, and Price $^{2}$ has advocated aspiration with a needle inserted into the anterior chamber to lower the intraocular pressure. In histological sections the air spaces above Descemet's membrane extend extremely close to the trabecular meshwork (which is anatomically continuous with Descemet's membrane). It is, therefore, possible that air gains access to the anterior chamber via the trabecular meshwork. If air had entered the anterior chamber through a rupture in Descemet's membrane one would have expected corneal oedema to be present in this area postoperatively, or the possibility of a 'double anterior chamber' with aqueous tracking into the lamellar graft bed. However neither of these complications was observed in our series 
suggesting that the air dissection did not rupture Descemet's membrane.

Final spectacle corrected vision in all four patients was better than $6 / 18$ and best corrected vision with a contact lens was $6 / 12$ or better. Whilst these visual results are less good than one would expect with penetrating keratoplasty they must be seen in the light of the reduced risks of lamellar keratoplasty. These include reduced risk of damage to anterior segment structures, the reduction in postoperative steroid treatment, and the lack of immunogenicity of lyophilised lenticules. This technique may thus be the treatment of choice where the aforementioned factors could be anticipated as problems if penetrating keratoplasty were to be undertaken, - for example, in badly vascularised corneas, pre-existing glaucoma or steroid sensitivity, or where there is the likelihood of poor compliance with follow-up.

In summary our experience with deep stromal air injection is that it makes lamellar keratoplasty an easier and safer operation to perform with acceptable visual outcome using either tissue cultured or lyophilised donor material. Further work is needed to improve the surgical technique and define how injected air reaches the anterior chamber, as it would be helpful to avoid this complication. The use of lyophilised tissue should theoretically avoid sensitisation of the eye and this may be beneficial if subsequent penetrating keratoplasty needs to be undertaken.
This paper was presented to the Ophthalmic Section of The Royal Society of Medicine on 13 June 1991.

1 Archila EA. Deep lamellar keratoplasty dissection of host issue with intrastromal air injection. Cornea 1984/1985; 3: 217-8.

2 Price FW. Air lamellar keratoplasty. Refract Corneal Surg $1989 ; 5: 240-3$.

3 McCulloch C, Thompson GA, Basu PK. Lamellar keratoplasty using full thickness donor material. Trans Am Ophthalmol Soc 1963; 61: 154-80.

4 Gasset AR. Lamellar keratoplasty in the treatment of keratoconus: conectomy. Ophthalmic Surg 1979; 10: 26-33.

5 Morrison JC, Swan KC. Full-thickness lamellar keratoplasty. A histologic study in human eyes. Ophthalmology 1982; 89: 715-9.

6 Jongebloed WL, Los LI, Worst JGF. Air-injection of the corneal stroma: a SEM study. Doc Ophthalmol 1989; 72 349-55.

7 Richards JM, Paton D, Gasset AR. A comparison of penetrating keratoplasty and lamellar keratoplasty in the surgical management of keratoconus. Am $\mathcal{F}$ Ophthalmol 1978; 86: 807-11.

8 Khodadoust AA. The allograft rejection reaction: the leading cause of late failure of clinical corneal grafts. Ciba Found Symp 1973; 15 (new series): 151-64.

9 Khodadoust AA, Silverstein AM. The survival and rejection of the epithelium in experimental corneal grafts. Invest the epithelium in experimental

10 Khodadoust AA, Silverstein AM. Transplantation and rejection of individual layers of the cornea. Invest Ophthalmol Vis Sci 1969; 8: 180-95.

11 Alldredge OC, Krachmer JH. Clinical types of corneal transplant rejection. Their manifestations, frequency, preoperative correlates, and treatment. Arch Ophthalmol 1981 99: 599-604.

12 Bourne WM. Morphologic and functional evaluation of the endothelium of transplanted human corneas. Trans Am Ophthalmol Soc 1983; 81: 403-50.

13 Laing RA, Neubauer L, Oak SS, Kayne HL, Leibowitz HM Evidence for mitosis in the adult corneal endothelium. Ophthalmology 1984; 91: 1129-34.

14 Rostron CK. Epikeratophakia: the living contact lens. Hospital Update 1987; 13: 819-29.

15 Frantz JM, Gebhardt BM, Reidy JJ, McDonald MB Immunogenicity of epikeratophakia tissue lenses containing living donor keratocytes. Refract Cormeal Surg 1991; 7: 141- 